\title{
Immunometabolism: Where Immunology and Metabolism Meet
}

\author{
Triantafyllos Chavakis \\ Institute for Clinical Chemistry and Laboratory Medicine, University Hospital and Faculty of Medicine Carl Gustav \\ Carus, TU Dresden, Dresden, Germany
}

\section{Keywords \\ Inflammation - Metabolism - Trained immunity . \\ Non-alcoholic fatty liver disease - Obesity - Adipose tissue . \\ Macrophages $\cdot$ Immunometabolism}

In the last two decades, "Immunometabolism" has emerged as a continuously increasing area of research with significant impact on biomedicine $[1,2]$. The term immunometabolism defines the interface between immunology and metabolism. The crosstalk between these two major research directions has multiple facets. For instance, immune cells present in the adipose tissue or liver regulate tissue homeostasis and metabolic functions, such as lipolysis or the actions of insulin [3-6]. Intake of nutrients, such as sugars, fat, or proteins, and the metabolism thereof may modulate immune responses [4]. Furthermore, the presence of chronic metabolic inflammation, also called "metaflammation" is a central hallmark of metabolic disorders [7]. Metabolic inflammation, orchestrated by cellular and molecular components of the immune response and affecting the adipose tissue, the liver, or the pancreas, contributes to pathogenesis and progression of conditions associated with obesity, such as

karger@karger.com www.karger.com/jin

Karger"
(C) 2021 The Author(s)

Published by S. Karger AG, Basel

This is an Open Access article licensed under the Creative Common Attribution-NonCommercial-4.0 International License (CC BY-NC) (http://www.karger.com/Services/OpenAccessLicense), applicable to the online version of the article only. Usage and distribution for commercial purposes requires written permission. type 2 diabetes or non-alcoholic fatty liver disease $[1,3,5$, 7]. For instance, both accumulation and activation of macrophages in the adipose tissue and the liver in obesity are implicated in the progression of metabolic disorders $[3,5]$. Interestingly, the function of macrophages in the adipose tissue and liver in obesity is regulated by adaptations of their metabolism, triggered by the lipid-rich tissue environment [3]. Accordingly, the obese adipose tissue and liver are characterized by the enrichment of a macrophage subpopulation with a lipid metabolism-related signature, designated "lipid-associated macrophages," as recently identified by analysis at the single-cell level $[8,9]$.

An important additional layer of the crosstalk between immunology and metabolism is illustrated by the fact that cellular metabolism is a central regulator of the function and activation of immune cells $[3,10]$. Distinct activation states of innate immune cells, especially macrophages, are associated with different cellular metabolic states; hence, cell metabolic adaptations facilitate macrophage phenotype and plasticity $[3,10]$. Metabolic reprogramming of innate immune cells is also an essential component of innate immune memory, also designated trained immunity, which defines that certain stimuli may promote unspe- 
cific memory in innate immune cells, exemplified by their increased inflammatory preparedness; trained innate immune cells exert higher inflammatory responses upon secondary challenges $[11,12]$. Cellular metabolism not only regulates the functions of mature myeloid cells, but also their generation in the bone marrow, a process termed myelopoiesis [13]. Adaptations in the metabolism of innate immune cell precursors, the hematopoietic stem and progenitor cells, in the bone marrow are integral to differentiation and lineage decisions in the context of myelopoiesis at steady-state and under conditions of stress [13].

The aforementioned examples of the multifaceted and continuously increasing field of immunometabolism illustrate the wide importance of the immunometabolic crosstalk in several pathologies. For instance, immunometabolic processes, including alterations in the metabolism of different immune cell populations, are integral constituents of the tumor microenvironment and hence influence tumor growth [14]. Additionally, immunometabolism has been implicated in autoimmune disorders, including type 1 diabetes and multiple sclerosis, as the loss of immunological tolerance and the accompanying emergence of autoreactive immune cells may be facilitated by specific alterations in the metabolism of immune cells [15]. Consistently, targeting the dysfunctional immunometabolic crosstalk represents a relevant approach for developing novel therapies not only for metabolic diseases but also for inflammatory disorders [2]. Along the same line, given the substantial contribution of innate immune cells, such as macrophages, to the hyperinflammatory response associated with severe COVID-19 disease, immunometabolic modulators could be potentially leveraged therapeutically for COVID-19 as well [16].

Due to the extraordinary relevance of the immunometabolism field and its major implications for health and disease, the current issue of the Journal of Innate Immunity includes a series of four review articles on this special topic. Two of the articles, written by Michailidou et al. and by Nati et al., review recent advances on the role of innate immune cells in the adipose tissue and liver, respectively $[17,18]$. Michailidou et al. review the role of macrophages, neutrophils, innate lymphoid cells, natural killer cells, and other innate immune cells in the adipose tissue in the lean state and in obesity and discuss the role of adipose tissue inflammation as well as its regulation by hypoxia in the context of obesity-associated metabolic disease [17]. Nati et al. discuss the role of neutrophils and macrophages in the liver in the context of non-alcoholic fatty liver disease [18]. The other two review articles by
Kolliniati et al. and Ferreira et al. focus, respectively, on the regulation of macrophage biology and the process of innate immune memory, by metabolic processes, such as glycolysis, the tricarboxylic acid cycle, lipid metabolism, or oxidative phosphorylation $[19,20]$. Kolliniati et al. review the impact of metabolic adaptations on macrophage plasticity and function in the context of different diseases, including cancer [20]. The article by Ferreira et al. provides insights on the important contribution of metabolic and epigenetic rewiring of innate immune cells in the context of trained immunity [19]. Importantly, all four review articles dedicated to the topic of immunometabolism provide relevant translational implications of how targeting immunometabolic processes may be leveraged in different pathologies and raise important open questions that should be addressed in future studies aiming at a better understanding of the intimate crosstalk between immunological and metabolic processes.

\section{Conflict of Interest Statement}

The author has no conflicts of interest to declare in relation to this work.

\section{Author Contributions}

T.C. wrote the manuscript.

\section{Funding Sources}

The research in the lab of T.C. is supported by funds from the European Research Council (ERC), the German Research Foundation (DFG), the National Institute of Dental and Craniofacial Research/National Institutes of Health (NIDCR/NIH), the German Ministry of Research and Education (BMBF), and the Else Kröner Fresenius Center for Digital Health Dresden.

\section{References}

1 Lee YS, Olefsky J. Chronic tissue inflammation and metabolic disease. Genes Dev. 2021 Mar;35(5-6):307-28.

2 Pålsson-McDermott EM, O’Neill LA. Targeting immunometabolism as an anti-inflammatory strategy. Cell Res. 2020 Apr;30(4):30014.

3 Daemen S, Schilling JD. The Interplay Between Tissue Niche and Macrophage Cellular Metabolism in Obesity. Front Immunol. 2020 Jan;10:3133.

4 Lee AH, Dixit VD. Dietary Regulation of Immunity. Immunity. 2020 Sep;53(3):510-23. 
5 Remmerie A, Martens L, Scott CL. Macrophage Subsets in Obesity, Aligning the Liver and Adipose Tissue. Front Endocrinol (Lausanne). 2020 Apr;11:259.

6 Trim WV, Lynch L. Immune and non-immune functions of adipose tissue leukocytes. Nat Rev Immunol. 2021 Nov. https://doi. org/10.1038/s41577-021-00635-7.

7 Hotamisligil GS. Inflammation, metaflammation and immunometabolic disorders. $\mathrm{Na}-$ ture. 2017 Feb;542(7640):177-85.

8 Daemen S, Gainullina A, Kalugotla G, He L, Chan MM, Beals JW, et al. Dynamic Shifts in the Composition of Resident and Recruited Macrophages Influence Tissue Remodeling in NASH. Cell Rep. 2021 Jan;34(2):108626.

9 Jaitin DA, Adlung L, Thaiss CA, Weiner A, Li B, Descamps H, et al. Lipid-Associated Macrophages Control Metabolic Homeostasis in a Trem2-Dependent Manner. Cell. 2019 Jul;178(3):686-698.e14.
10 Ryan DG, O’Neill LA. Krebs Cycle Reborn in Macrophage Immunometabolism. Annu Rev Immunol. 2020 Apr;38(1):289-313.

11 Bekkering S, Domínguez-Andrés J, Joosten LA, Riksen NP, Netea MG. Trained Immunity: Reprogramming Innate Immunity in Health and Disease. Annu Rev Immunol. 2021 Apr;39(1):667-93.

12 Penkov S, Mitroulis I, Hajishengallis G, Chavakis T. Immunometabolic Crosstalk: An Ancestral Principle of Trained Immunity? Trends Immunol. 2019 Jan;40(1):1-11.

13 Hajishengallis G, Li X, Chavakis T. Immunometabolic control of hematopoiesis. Mol Aspects Med. 2021 Feb;77:100923.

14 Kaymak I, Williams KS, Cantor JR, Jones RG. Immunometabolic Interplay in the Tumor Microenvironment. Cancer Cell. 2021 Jan;39(1):28-37.

15 Galgani M, Bruzzaniti S, Matarese G. Immunometabolism and autoimmunity. Curr Opin Immunol. 2020 Dec;67:10-7.
16 O'Carroll SM, O'Neill LAJ. Targeting immunometabolism to treat COVID-19. Immunother Adv. 2021 Jan;1(1):Itab013.

17 Michailidou Z, Gomez-Salazar M, Alexaki VI. Innate Immune Cells in the Adipose Tissue in Health and Metabolic Disease. J Innate Immun. 2021 Apr;(Apr):1-27.

18 Nati M, Chung KJ, Chavakis T. The Role of Innate Immune Cells in Nonalcoholic Fatty Liver Disease. J Innate Immun. 2021 Aug;(Aug):1-11.

19 Ferreira AV, Domiguéz-Andrés J, Netea MG. The Role of Cell Metabolism in Innate Immune Memory. J Innate Immun. 2020 Dec;(Dec):1-9.

20 Kolliniati O, Ieronymaki E, Vergadi E, Tsatsanis C. Metabolic Regulation of Macrophage Activation. J Innate Immun. 2021 Jul;(Jul):1-17. 